
\title{
28 Research Suare \\ Effects of auriculotherapy for postoperative nausea and vomiting: A systematic review protocol
}

Isabella Boechat Faria Santos ( $\nabla$ isabella.boechatfs@gmail.com )

Universidade Federal do Pará: Universidade Federal do Para https://orcid.org/0000-0001-8312-8752

Amanda Suzane Alves da Silva

Universidade Federal do Pará: Universidade Federal do Para

Giovana Salomão Melo

UFPA: Universidade Federal do Para

Cleuma Oliveira Soares

UFPA: Universidade Federal do Para

Edila Monteiro de Andrade

UFPA: Universidade Federal do Para

Lúcio Flávio Garcia Rodrigues

UFPA: Universidade Federal do Para

Thalia Saraiva Mendonça

UFPA: Universidade Federal do Para

\section{Diego Bessa Dantas}

UEL: Universidade Estadual de Londrina

João Simão de Melo-Neto

UFPA: Universidade Federal do Para

\section{Protocol}

Keywords: Acupuncture, Ear, Acupressure, Antiemetics, Postoperative Nausea and Vomiting, Surgery

Posted Date: November 23rd, 2021

DOI: https://doi.org/10.21203/rs.3.rs-1090857/v1

License: (c) (1) This work is licensed under a Creative Commons Attribution 4.0 International License.

Read Full License 


\section{Abstract \\ Background}

Postoperative nausea and vomiting (PONV) is a distressing complication of anesthesia and can lead to aspiration, dehydration, and electrolyte imbalance. Antiemetic agents are conventionally prescribed to manage PONV; however, they have associated side effects. Therefore, unconventional methods, such as auricular acupuncture (AA), are also utilized to prevent and control emesis after surgery. AA originated in traditional Chinese medicine and is based on a diagnostic and treatment system that aims to normalize dysfunction through stimulation of reflex points on the ear. The aim of this study is to evaluate the effects of AA in controlling PONV.

\section{Methods}

We will perform a systematic review according to the Cochrane methodology. An overall search strategy will be developed and adapted for PubMed, PEDro, the Virtual Health Library, SciELO, EMBASE, the Web of Science, SciVerse Scopus, and the Cochrane Library to search for the following descriptors: "Acupressure"; "Antiemetics"; "Postoperative nausea and vomiting"; "Surgery"; "Auriculotherapy"; "Nausea"; "Vomiting"; and "Postoperative period." Articles with a mean score of $6 \pm 1.5$ on the PEDro scale will be evaluated. The size of the intervention effect (Z) will be calculated for each outcome included in this review. The primary outcome will be the incidence of PONV. The secondary outcome will be the severity of PONV. Quality assessment will be performed with the Cochrane instrument. If possible, a meta-analysis will be performed using Review Manager 5.3 software.

\section{Discussion}

Several studies have reported positive outcomes of AA for patients with PONV. This study could provide robust and conclusive evidence of the usefulness of $A A$ as an effective treatment alternative for emesis without the side effects of conventional medication.

\section{Trial registration}

Systematic review registration number: CRD42020149772 (S1 File)

\section{Background}

In the postoperative period of surgical intervention under anesthesia, emesis is a side effect, characterized by nausea and vomiting $[1,2]$. The incidences of nausea and vomiting after surgery under anesthesia are $50 \%$ and $30 \%$, respectively [3]. Patient-controlled analgesics, such as morphine, provide pain relief but can also induce postoperative nausea and vomiting (PONV). Emesis can lead to aspiration, 
dehydration, and electrolyte imbalance [4], thereby compromising the patient's quality of life and adherence to treatment and increasing the length of hospital stay, hindering the return to activities of daily living [1, 2].

Drugs to treat emesis act on type 3 serotonin, muscarinic, type 2 dopamine, and opioid receptors [5]. Antiemetic medications can elevate blood glucose levels [6] and cause other adverse effects, such as hypotension, hypertension, dysphoria, hallucinations, excessive sedation, dry mouth, psychological disturbances, or pyramidal symptoms $[7,8]$. Therefore, there is a need for alternative methods, such as auricular acupuncture, that have fewer side effects are aimed at replacing antiemetic medications [9].

In the past four decades, acupuncture has become widespread at occidental health care centers. For pain relief, scientific studies have shown acupuncture to be effective. Acupuncture is an old medical treatment that originated in China [8], and auricular acupuncture is a subtype that involves stimulation of ear acupoints. There are 93 specific points identified for auriculotherapy, which are located in and around the ear. These points can be subjected to needle stimulation, ear acupressure with seeds or pallets, laser stimulation, or electrostimulation [10]. The World Health Organization has presented a list of medical conditions for which acupuncture can have positive results, including the prevention and treatment of PONV [11]. However, the effects of this technique on postoperative patients have not been fully elucidated.

Since emesis is a common side effect after surgery and auricular acupuncture is an alternative treatment method, the aim of this study is to review the effects of this technique on the control of PONV.

\section{Methods/design}

\section{Type of study}

We will conduct a systematic review of the literature with meta-analysis (if possible) on the effects of complementary therapy in the reduction of nausea and vomiting in the study population based on the PRISMA Statement recommendations [12] (S2 File). This study is directly related to human health.

\section{Eligibility criteria}

Available data will be searched in relevant international journals in English, Portuguese, Spanish, and Mandarin with previously defined criteria according to the PICOS acronym (Population, Intervention, Comparison, Outcome, and Study type).

\section{Population}

Patients in the early postoperative period after surgical intervention under anesthesia who presented with emesis (nausea and vomiting) will be included in this systematic review.

\section{Intervention}


Studies that used an ear acupoint acupuncture to reduce or control emesis in patients after surgical interventions will be analyzed. Applications of the following types will be considered: transcutaneous electrical nerve stimulation, needles, seeds or pallets, and laser electrostimulation.

\section{Comparison}

Only studies that compared an experimental group with a control group of placebo or other interventions will be included.

\section{Outcomes}

\section{Primary outcomes}

The primary outcome will be the occurrence of nausea and vomiting in the postoperative period. Emesis will be considered in the absence of distinction between nausea and vomiting.

\section{Secondary outcomes}

The secondary outcome will be the severity of PONV.

\section{Search strategy}

The search terms for the research databases, selected from Medical Subject Headings and Health Sciences descriptors, are as follows: "Acupressure"; "Antiemetics"; "Postoperative nausea and vomiting"; "Surgery"; "Auriculotherapy"; "Nausea"; "Vomiting"; and "Postoperative period." A search strategy model will be developed for PubMed, PEDro, the Virtual Health Library, SciELO, EMBASE, the Web of Science, SciVerse Scopus, and the Cochrane Library. Studies published every year up to 2021 will be searched.

\section{Study selection}

For the initial selection, studies that do not address the topic will be excluded. Two reviewers will perform a secondary selection to eliminate irrelevant studies. The order of the review will be as follows: title, abstract, and full reading (Fig.1). All studies potentially eligible for inclusion in the review will be selected for full reading. In cases of disagreement, a third reviewer will be consulted.

Figure 1. Study Selection for Review.

\section{Study quality assessment}

The qualification of the methodology used in the research will be analyzed using PEDro.

\section{Selected information}

Eligible studies will undergo a data extraction and management step. A standardized form will be used to guide the extraction of the following information: population, intervention group (acupoints used), 
comparison group (proposed protocol), outcome, and type of study. Studies that do not fully meet the inclusion criteria will be excluded and tabulated with their justification for the exclusion shown in table 1 . The characteristics of the selected studies will be presented as shown in table 2 .

Table 1. Study characteristics related to the number of participants, inclusion, and exclusion criteria.

\begin{tabular}{|ll|}
\hline Author / Year No. of participants Inclusion criteria Exclusion criteria \\
\hline
\end{tabular}

Table 2. Study Characteristics related to the configuration.

\begin{tabular}{|llllllll|}
\hline Title / & $\begin{array}{l}\text { Signs and } \\
\text { Symptoms }\end{array}$ & $\begin{array}{l}\text { No. of } \\
\text { participant } \\
\text { per group }\end{array}$ & $\begin{array}{l}\text { Definition } \\
\text { of Auriculo } \\
\text { Year }\end{array}$ & $\begin{array}{l}\text { Comparison } \\
\text { therapy } \\
\text { (protocol } \\
\text { details) }\end{array}$ & Outcome & Results & PEDro \\
\hline & & & & & \\
& & & & & & \\
\end{tabular}

The methodological quality, risk of bias, and statistical report will be assessed on the PEDro scale for the risk of bias using the following parameters: concealment of allocation, allocation generation, masking (of participants and researchers), the presence of incomplete data, and reporting biases of information or other types. The answers to these domains can be "Yes," "No," or "Where" [13]. The score will only be awarded when a criterion is clearly satisfied. Moreover, the recommendations of the Preferred Report Items for Systematic Reviews and PRISMA statement will be followed [14].

Rayyan is a web-enabled application that helps authors of systematic reviews optimize the study development process. The software helps include and exclude articles using the abstract, title, filters, keywords, authors, year of publication, and label. Another advantage of Rayyan is the ability to have multiple blind collaborators work on the same review. In this study, three reviewers will be used for the impartial selection of articles [15].

Results regarding the study population will be presented as descriptive. The size of the intervention effect (Z) will be calculated for each study included in this review using the mean difference or standardized mean difference. For data with low heterogeneity, a meta-analysis of fixed effects will be performed to estimate the effect of treatment on patients with PONV. For data with high heterogeneity, a randomeffects model will be utilized. The absence of publication bias will be analyzed using a funnel plot. $P$ value $<0.05$ will be set as statistical significance. Review Manager software will be used for all analyses, including the meta-analysis if possible [16]. 


\section{Discussion}

Previous studies have shown auriculotherapy as an alternative or complementary option for antiemetics to manage PONV. Nevertheless, there are controversies and a lack of consensus in the literature regarding the methodologies and results of this therapy.

Although there are currently several study formats in the literature related to the understanding of the auricular acupuncture effects in postoperative emesis (whether qualitative or quantitative), this study design seeks to highlight the main points of randomized and nonrandomized clinical trials, thus evaluating the available evidence with better methodological precision. This systematic review (and meta-analysis, if feasible) is aimed at systematizing the various effects and possible results of this technique.

This study could provide clear and robust evidence regarding the usefulness of ear acupuncture to treat postoperative emesis in addition to a consensus of methodology that would warrant the success of this alternative therapy. Further, it could pave the way for high-quality future studies on the subject.

\section{Limitations}

This systematic review with meta-analysis may be limited if there is a scarcity of studies in the literature, heterogeneity of experimental protocols and absence of data from studies for analysis, methodological quality of the studies.

\section{Abbreviations}

PONV - Postoperative nausea and vomiting

AA - Auricular acupuncture

$Z$ - The size of the intervention effect

\section{Declarations}

\section{Ethics approval and consent to participate}

Not applicable

\section{Consent for publication}

Not applicable

\section{Availability of data and materials}

Not applicable 


\section{Competing interests}

The authors declare that there are no conflicts of interest.

\section{Funding}

Not applicable

\section{Authors' contributions}

All authors contributed equally to this work and approved the submitted version.

IBFS writing original draft; data curation; visualization; methodology; validation; writing review \& editing; ASAS writing - original draft; data curation; visualization; writing - review \& editing; GSM writing - original draft; data curation; visualization; writing - review \& editing; COS writing - original draft; data curation; visualization; writing - review \& editing; EMA writing - original draft; data curation; visualization; writing review \& editing; LFGR writing - original draft; data curation; visualization; writing - review \& editing; TSM writing - original draft; data curation; visualization; writing - review \& editing; DBD writing - original draft; writing - review \& editing; JSMN formal analysis, writing - review \& editing, supervision.

\section{Acknowledgements}

We thank the clinical and experimental research units of the urogenital system (UPCEURG) of UFPA

\section{References}

1 Kapur PA. The big "little problem". Anesth Analg. 1991;73(3):243-245.

2 Kim Y, Kim CW, Kim KS. Clinical observations on postoperative vomiting treated by auricular acupuncture. Am J Chin Med. 2003;31(3):475-480.

3 Motamed C, Bourgain JL. Postoperative nausea and vomiting in the post-anesthetic care unit, a 5-year survey of a quality assurance program in surgical cancer patients. Bull Cancer. 2015;102(5):405-410.

4 Yeh ML, Tsou MEU, Lee BY, Chen HH, Chung YC. Effects of Auricular Acupressure on Pain Reduction in Patient-controlled Analgesia After Lumbar Spine Surgery. Acta Anaesthesiol Taiwan. 2010;48(2):80-86.

5 Öbrink E, Jildenstål P, Oddby E, Jakobsson JG. Post-operative nausea and vomiting: update on predicting the probability and ways to minimize its occurrence, with focus on ambulatory surgery. Int $\mathrm{J}$ Surg. 2015;15:100-106.

6 Yang T, Liu Q, Lu M, Ma L, Zhou Y, Cui Y. Efficacy of olanzapine for the prophylaxis of chemotherapyinduced nausea and vomiting: a meta-analysis. Br J Clin Pharmacol. 2017;83 (7):1369-1379. 
7 Firouzian A, Kiasari AZ, Godazandeh G, Baradari AG, Alipour A, Taheri A, Emami Zeydi A, Montazemi A. The effect of intravenous dextrose administration for prevention of post-operative nausea and vomiting after laparoscopic cholecystectomy: A double-blind, randomised controlled trial. Indian J Anaesth. 2017;61(10):803-810.

8 Hao JJ, Mittelman M. Acupuncture: past, present, and future. Glob Adv Health Med. 2014;3(4):6-8.

9 Feng C, Popovic J, Kline R, Kim J, Matos R, Lee S, Bosco J. Auricular Acupressure in the Prevention of Postoperative Nausea and Emesis A Randomized Controlled Trial. Bull Hosp Jt Dis (2013).2017;75(2):114-118.

10 Zhang CS, Yang AW, Zhang AL, May BH, Xue CC. Sham Control Methods Used in Ear-Acupuncture/EarAcupressure Randomized Controlled Trials: A Systematic Review. J Altern Complement Med. 2014;20(3):147-161.

11 NIH Consensus Conference. Acupuncture. JAMA. 1998;280(17):1518-1524.

12 Liberati A, Altman DG, Tetzlaff J, Mulrow C, Gotzche PC, loannidis JP, Clarke M, Devereaux PJ, Kleijnen J, Moher D. The PRISMA Statement for Reporting Systematic Reviews and Meta-Analyses of Studies That Evaluate Health Care Interventions: explanation and elaboration. PLoS Med. 2009;6(7).

13 Physiotherapy Evidence Database [https://www.pedro.org.au/english/downloads/pedro-scale/]. Available in: https://www.pedro.org.au/[cited 10 December 2020].

14 Moher D, Shamseer L, Clarke M, Ghersi D, Liberati A, Petticrew M, et al. Preferred reporting items for systematic review and meta-analysis protocols (PRISMA-P) statement. Systematic Reviews 4.1(2015):29.

15 Ouzzani M, Hammady H, Fedorowicz Z, et al. Rayyan-a web and mobile app for systematic reviews. Syst Rev 5, 210 (2016). DOI:10.1186/s13643-016-0384-4.

16 Review Manager (RevMan) [Computer program]. Version 5.3. Copenhagen: The Nordic Cochrane Centre, The Cochrane Collaboration, 2014.

\section{Figures}


Santos IBF, Silva ASA, Melo GS, Soares CO, Andrade EM, Rodrigues LFG, Mendonça TS, Dantas DB, Melo-Neto JS. Effects of auriculotherapy for postoperative nausea and vomiting: A systematic review protocol

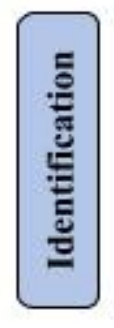

Records identified in the search for the bases Pubmed, PEdro, Virtual Health Library, Scielo, Embase, Web of science, SciVerse Scopus and Cochrane Central
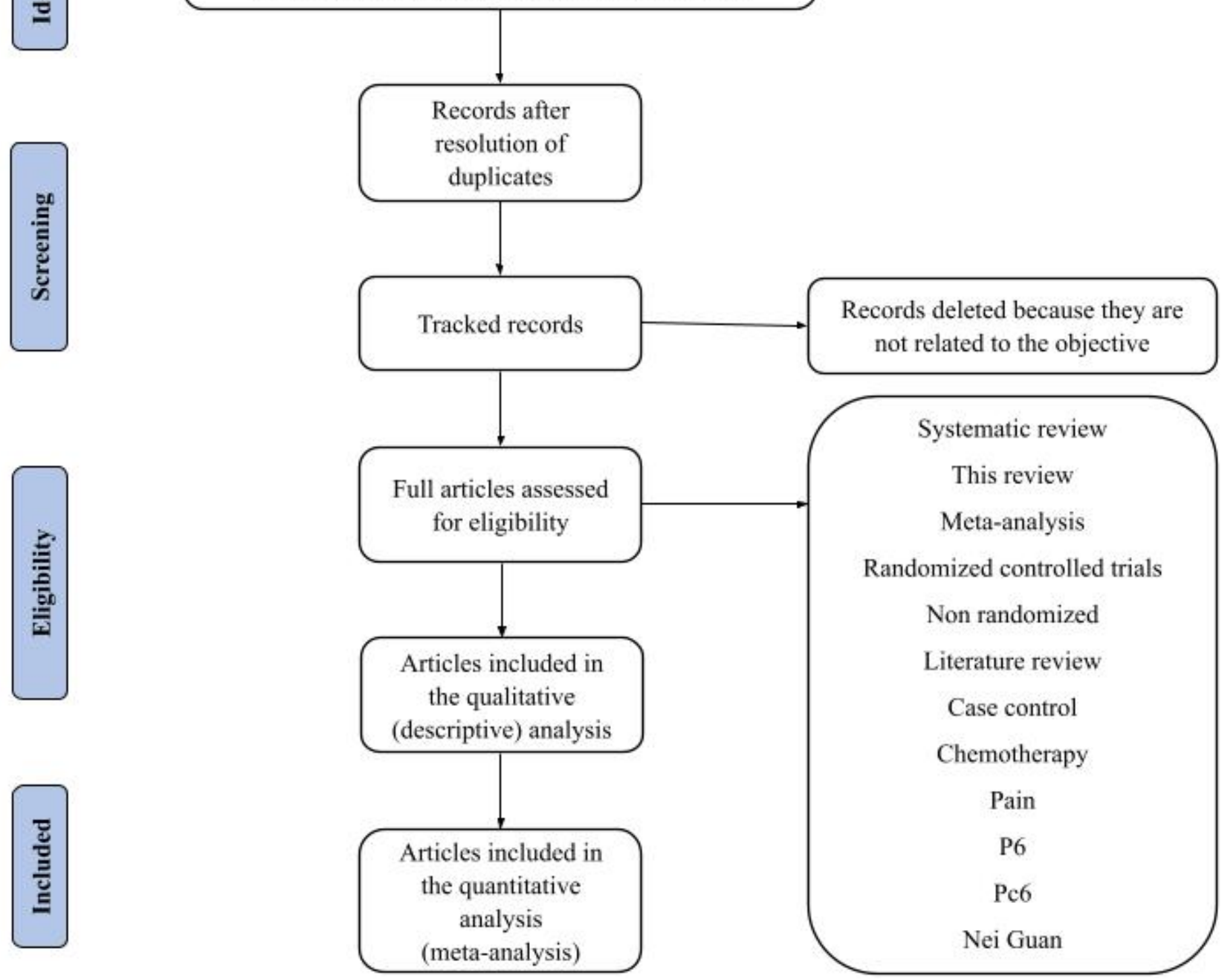

Figure 1

Study Selection for Review.

\section{Supplementary Files}

This is a list of supplementary files associated with this preprint. Click to download.

- S1File.docx 
- S2File.pdf

Page 10/10 